\title{
The impact of Anopheles gambiae egg storage for mass rearing and production success
}

\author{
Ernest Mazigo ${ }^{1}$, Winifrida Kidima' ${ }^{1}$ Joseph Myamba² and Eliningaya J. Kweka ${ }^{3,4^{*}}$ (i)
}

\begin{abstract}
Background: Mass rearing requires a large colony from which male individuals can be harvested for sterilization and release. Attention is needed when monitoring life parameters of the reared population, knowing that any variations within the target population would lead to mismatching between two populations. The aim of this study was to assess the impact of Anopheles gambiae sensu stricto (s.s.) egg storage on hatchability and life history traits. For each parameter, comparison was made between freshly laid and stored eggs in three densities (40, 80, 120 eggs).
\end{abstract}

Methods: Anopheles gambiae s.s. freshly laid eggs were collected from the Tropical Pesticide Research Institute (TPRI) insectary. Eggs to be stored were kept at $-20^{\circ} \mathrm{C}$ for $10 \mathrm{~min}$ and then transferred to refrigerators at $4{ }^{\circ} \mathrm{C}$ for intervals of $5,10,15,20$, and 25 days. After respective storage days, the eggs were transferred from refrigerators to ambient temperature of $(25 \pm 2)^{\circ} \mathrm{C}$ for $24 \mathrm{~h}$ and then placed in incubators for $24 \mathrm{~h}$. Thereafter eggs were hatched. The egg hatchability, emerged larvae development, larvae survival and emerged adult sex ratios were monitored.

Results: This study found that hatching rates decreased with increase in storage time. The difference was significant in eggs stored for 10 and 15 days $(P<0.05)$. There were no significant differences in hatching rates between An. gambiae eggs stored for 5 days and freshly hatched eggs $(P>0.05)$. Anopheles larvae development (L1 to pupae) was not significantly affected by storage time across all hatching densities. The study also found that larvae survival decreased with increase in egg storage time. However, there was no significant difference between larvae from freshly hatched eggs and those from eggs at 5 and 10 storage days $(P>0.05)$ but not for eggs stored for 15 days. Furthermore, there was a decrease in emerged adult males and increase in females relative to increased time of egg storage. The difference was significant $(P<0.05)$ at 15 storage days but not for eggs stored for 5 and 10 days (in triplicate densities).

Conclusion: From this study it was concluded that storing An. gambiae eggs at $4{ }^{\circ} \mathrm{C}$ and $48 \pm 2 \%$ relative humidity $(\mathrm{RH})$ for 5 days is the optimal condition and time that did not affect egg hatching rates, larval development and survivorship and emerged adult mosquito sex ratio.

Keywords: Mass rearing, Anopheles gambiae, Plasmodium falciparum, Sterile insect technique, Hatch rate index, Life history traits

\section{Background}

Anopheles gambiae is a group comprised of eight members that are morphologically indistinguishable sibling species of mosquitoes in the genus Anopheles namely Anopheles melas, Anopheles merus, Anopheles arabiensis, Anopheles bwambwae, Anopheles coluzzii, Anopheles

\footnotetext{
*Correspondence: eliningaya.kweka@tpri.go.tz

${ }^{4}$ Division of Livestock and Human Disease Vector Control, Tropical

Pesticides Research Institute, P.O.Box 3024, Arusha, Tanzania

Full list of author information is available at the end of the article
}

quadriannulatus, Anopheles ahimaricus and Anopheles gambiae sensu stricto (s.s.) [1]. Among these, An. gambiae s.s., An. coluzzii and An. arabiensis are the most efficient malaria vectors in the sub-Saharan region $[1,2]$. Malaria prevalence is high, keeping at risk more than 3.2 billion worldwide, $92 \%$ in the sub-Saharan Africa region where 10 countries have high malaria transmission based on the 2018 World Health Organization (WHO) malaria report [3]. However, malaria vector population decline has been reported from several parts of Africa where transmission was recorded to be higher before

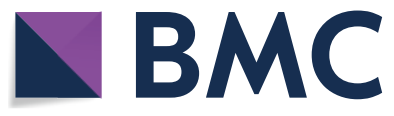

(c) The Author(s) 2019. This article is distributed under the terms of the Creative Commons Attribution 4.0 International License (http://creativecommons.org/licenses/by/4.0/), which permits unrestricted use, distribution, and reproduction in any medium, provided you give appropriate credit to the original author(s) and the source, provide a link to the Creative Commons license, and indicate if changes were made. The Creative Commons Public Domain Dedication waiver (http://creativecommons.org/ publicdomain/zero/1.0/) applies to the data made available in this article, unless otherwise stated. 
installation of various intervention tools implementation [4-7].

The use of long-lasting insecticide-treated nets (LLINs), indoor residual spraying (IRS), improved diagnosis, and treatment with artemisinin-based combination therapy (ACT) has contributed to the decrease in malaria burden in endemic areas [7, 8]. For instance, the 2016 global malaria burden report presented a worldwide decline of mortality rates from 2010 by $38 \%$, with a $42 \%$ decrease reported in Africa [9]. The data show promising trends towards the WHO strategy for elimination of malaria by 2015-2030 [10]. Unfortunately intensive use of insecticides has led to the vector reduced susceptibility status in various parts of Africa [11-15]. Apart from insecticide resistance, behavioural changes recorded in some places have been recognised as a type of insecticide resistance [14]. For example, malaria vectors exhibit some behavioural changes to avoid intra-domiciliary control tools [16-18]. Such behavioural changes include a tendency to bite in the early hours of the night or at dawn, which circumvents current control strategies $[16,19]$. As proposed by the World Health Malaria Control Programme, elimination of malaria in African endemic areas by 2030 will not be possible if these challenges are not met $[9,10,20]$. There is the necessity to establish alternative vector control strategies: sterile insect technique might be one of them [21]. Sterile insect technique is a biologically based method to control disease vectors through the introduction of sterile males into the target population [22].

Sterile insect technique is a novel intervention requiring mass rearing of insects under laboratory conditions, exposing the males to gamma rays to induce sexual sterility before release into a target population [22]. Mosquito eggs are known to be the only stage at which diapauses to embryo development can be induced by certain environmental conditions, allowing storage for specific intervals of time without affecting egg viability and other life parameters [23]. Mass rearing of mosquitoes requires large number of eggs from which an experimental population would be harvested [24]. Studies on viability of Anopheles eggs showed that eggs hatch within 2 days post-oviposition under optimal environmental conditions $\left(27{ }^{\circ} \mathrm{C} \pm 2,80 \% \pm 10\right.$ relative humidity $(\mathrm{RH})[25,26]$. However, changes in environmental conditions have been shown to affect egg viability and induce delayed hatching in mosquitoes [27]. In the laboratory, Anopheles eggs can be preserved in different conditions with some resultant certainties of their viability, developmental parameters and emerged adult sex ratios (females emerged against total mosquitoes (males and females emerged)) [23, 28]. There is a need to establish specified laboratory conditions under which eggs would be handled and stored for optimal breeding output of mosquitoes with traits similar to that of the wild population for effective insect sterile technique. This study aims at investigating the impact of laboratory storage conditions, particularly temperature, on hatching rates of An. gambiae eggs and associated life history traits.

\section{Methods}

\section{Study area and study design}

The study was conducted in the Livestock and Human Diseases Vectors Division, Tropical Pesticide Research Institute (TPRI), Arusha. The institution insectaries have An. gambiae s.s. Kisumu strain (R 70) established since 1992 with a colony from Kisumu, Kenya. The insectary is equipped with necessary instruments for managing and rearing mosquitoes. Such instruments include humidifiers, electric heaters, 12:12 h synchronized lighting schedule, thermometers and hygrometers.

Anopheles gambiae freshly laid eggs were collected from the TPRI insectary. A total of 30 filter papers, each with more than 480 eggs, were used for the complete study. A single experiment used 5 filter papers (with more than 2400 eggs). A total of 6 experiments were carried out in triplicate densities (40, 80 and 120 eggs). A total of 14,400 eggs were used for the complete study (=30 hatching filter papers).

For a single experiment 5 hatching filter papers were randomized into five groups of storage days: the first filter paper was stored for 5 days, the second for 10, the third for 15, the fourth for 20 , and the fifth for 25 days. Each filter paper (with about 480 eggs), was divided into two parts containing equal number of eggs $(n=240)$ : one portion was used as control and the other $(n=240)$ for experiment. The control $(n=240)$ was further subdivided into three different densities $(\mathrm{n}=40, \mathrm{n}=80$, $\mathrm{n}=120$ ). Each was then hatched separately into hatching plates of $15 \times 10 \times 8 \mathrm{~cm}$ in size with $500 \mathrm{ml}$ of tap water placed under standard conditions of temperature $(27 \pm 1){ }^{\circ} \mathrm{C}$ and relative humidity $(80 \pm 10) \%$ [29]. The experimental filter papers (with 240 eggs) were stored following procedures described below.

\section{Procedures for storing Anopheles gambiae eggs}

Each filter paper containing 240 eggs was placed separately on top of wet cotton wool in different $100 \times 15 \mathrm{~mm}$ petri dishes. For a single experiment, a total of 5 petri dishes each with 240 enclosed eggs was used. The 5 petri dishes were covered with a cap, tightened with masking tape and then randomized to different storage days $(5,10$, 15,20 , and 25 days). The petri dishes were then placed into deep freezers at $-20{ }^{\circ} \mathrm{C}$ for $10 \mathrm{~min}$ to heat-shock 
and stop embryo development before being transferred into refrigerators for storage at $4{ }^{\circ} \mathrm{C}$ and $48 \pm 2 \% \mathrm{RH}$. Petri dishes were then removed from the refrigerators after respective required storage days. After refrigeration, the petri dishes were immediately placed at ambient temperature $(25 \pm 2){ }^{\circ} \mathrm{C}$, and $78 \pm 2 \% \mathrm{RH}$ for $24 \mathrm{~h}$. Thereafter, the petri dishes were transferred into an incubator at $(27 \pm 2){ }^{\circ} \mathrm{C},(90 \pm 10) \% \mathrm{RH}$ for another $24 \mathrm{~h}$. After this, filter papers with eggs were removed from petri dishes and eggs were counted manually using hand lens and counter machine. The eggs were placed into hatching plates of $15 \times 10 \times 8 \mathrm{~cm}$ in size, filled with $500 \mathrm{ml}$ of tap water in triplicate egg densities of 40, 80 and 120. Eggs were hatched at $27 \pm 1{ }^{\circ} \mathrm{C}$ and $80 \pm 10 \% \mathrm{RH}$. The experiments were repeated six times.

\section{Impact of Anopheles gambiae egg storage time on hatching rates}

Hatching rates were monitored twice per day (08:00 and $18.00 \mathrm{~h}$ ) from when the eggs were washed from filter paper to rearing plate after $24 \mathrm{~h}$ of incubation. For each single experiment, the record of eggs hatched was obtained by counting first-instar larvae (F1) within recipient hatching plates. The total $\mathrm{F} 1$ in recipient plates was considered equal to the number of viable eggs per density.

\section{Impact of Anopheles gambiae egg storage time on larval development}

Emerged larvae were daily fed with Tetramin fish food (Tetra GmbH, Herrenteich 78, 49324 Melle, expiring March 2019) at the rate of 0.0003 gm per larval [30]. Rearing temperature and humidity were regulated and maintained at $28 \pm 2{ }^{\circ} \mathrm{C}$ and $78 \pm 2 \% \mathrm{RH}$ (Humidifiers, electric heaters and probe type T, Digitron Thermometer Instruments were used) [29].

Mosquito larvae size and larval developmental were used to monitor variations in larval development. Larvae developmental records were taken twice per day (08:00 and 18:00) after which the days on which all larvae in respective densities pupated or emerged into adults were analysed. Dead larvae were recorded and removed from plates using plastic pipettes. Pupae were put into paper cups $(70 \mathrm{~cm}$ internal diameter with $100 \mathrm{ml}$ of dichlorinated water) and transferred to rearing cage $(30 \times 30 \times 30 \mathrm{~cm})$ for adult emergence, at $27 \pm 2{ }^{\circ} \mathrm{C}$ and $78 \pm 2 \% \mathrm{RH}$. Larval development was recorded according to hatching densities and storage duration. Larval developmental time at different hatching densities and storage days were estimated as the average duration from first-instar larval hatching to emergence of adult mosquitoes.

\section{Impact of Anopheles gambiae egg storage time on larvae survivorship}

Live and dead larvae were counted, after which the dead were removed from rearing plates. Survival rates of $A n$. gambiae larvae were calculated as proportional ratio of daily larval count at a given stage to the total hatched eggs of that density. Proportion computations were separated into densities and egg storage days.

\section{Impact of Anopheles gambiae egg storage time on adult sex ratio}

Emerged adults were fed with $10 \%$ sucrose sugar, removed from cages using mouth aspirator at age 6-7 days postemergence. Antennae and mouth parts were used to sort them into sex. Adult sex ratios were computed as the number of emerged adult females divided by the total number of emerged adults (males and females). Data were recorded according to egg hatching density and egg storage days.

\section{Statistical analyses}

Data obtained were cleaned for layouts using MS-Excel. Analysis was done by using Graph Pad Prism software version 7.04 (Graph Pad-Prism Software, Inc. Oxford University 2017). Data were tested for normality using Shapiro-Wilk test. Study results were descriptively summarized using frequencies, proportions, means and standard deviations. Daily hatch computations were done for every $24 \mathrm{~h}$. Hatch records were separated into triplicate densities (40, 80 and 120 eggs) and storage time. Comparison of hatching rates, larval development and sex ratios between stored and freshly laid eggs were analysed using paired samples t-test. The Kaplan-Meier survival analysis was done to determine the effect of storage time on larval survivorship proportions against freshly hatched eggs in similar densities. Adult sex ratios and larval survivorship were calculated by proportional tests. Associated charts and graphs were done using Microsoft Excel 2007 (Microsoft, WA, USA).

\section{Results}

Impact of egg storage time on hatching rates of Anopheles gambiae

Higher mean hatch rates were observed on eggs stored for 5 days. There was an abrupt decrease in hatching rates for eggs stored for 10 and 15 days (Fig. 1b, c). About $93 \%$ of eggs stored for 5 days at densities of 40, 80 and 120 hatched successfully. Hatching rates between eggs stored for 5 days and freshly laid eggs at 40, 80 and 120 densities were not significantly different (Fig. 1a, Table 1). Percentage hatch rates of eggs stored for 10 days at 40, 80 and 120 densities were above $84 \%$ although they were significantly lower than hatching rates of freshly laid eggs at 


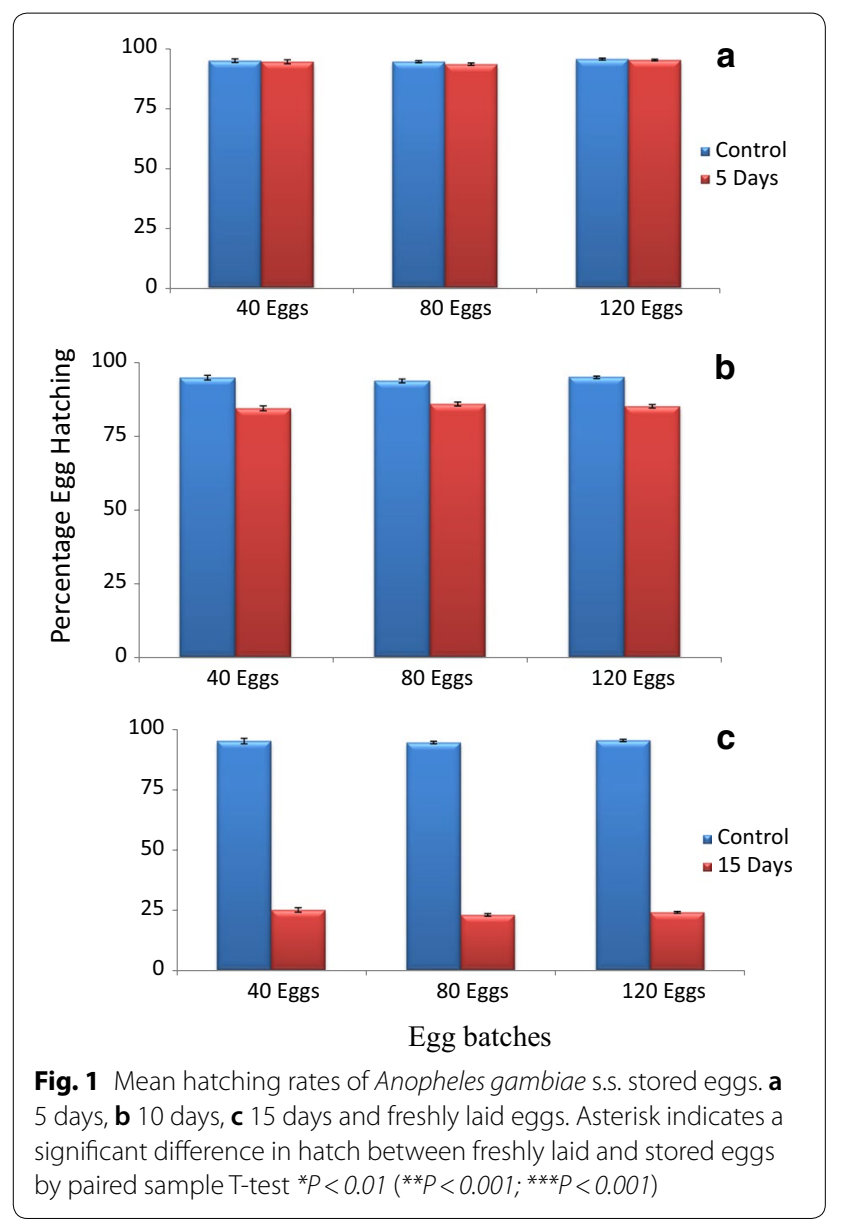

Table 1 of the mean hatching rates between freshly hatched and stored An. gambiae s.s. eggs at different densities

\begin{tabular}{llll}
\hline Density & \multicolumn{3}{l}{$\begin{array}{l}\text { Freshly hatched and stored eggs hatched at different } \\
\text { densities }\end{array}$} \\
\cline { 2 - 4 } & $\mathbf{5}$ days & 10 days & $\mathbf{1 5}$ days \\
\hline 40 eggs & $t=0.31, P=0.77$ & $t=8.73, P<0.01$ & $t=62.61, P<0.001$ \\
80 eggs & $t=2.07, P=0.09$ & $t=11.35, P<0.01$ & $t=76.32, P<0.001$ \\
120 eggs & $t=0.72, P=0.49$ & $t=14.69, P<0.01$ & $t=250.32, P=0.001$ \\
\hline
\end{tabular}

similar densities (Fig. 1b, Table 1). Eggs stored for 15 days had hatching rates below 29\% and significantly lower than those for freshly laid eggs (Fig. 1c, Table 1). Eggs stored for 20 and 25 days did not hatch.

\section{Larval development between similar hatching densities of freshly laid and stored Anopheles gambiae eggs}

Development time increased with increase in storage time. Larvae from eggs stored for 15 days took more days to pupate than those from days 5 and 10 . Overall larvae from stored eggs took longer to pupate than those from freshly laid eggs although the difference was not significant at 5, 10 and 15 storage days in the triplicate densities (Table 2).

\section{Impact of An. gambiae egg storage time on larval survivorship at different densities}

Proportions of larvae surviving to adult stage decreased with increase in egg storage time (Table 3). Survival rates of larvae from both freshly laid (control) and stored eggs (experimental) dropped after day 2 of hatching, with mortality rate being more pronounced in larvae from stored eggs (Figs. 2, 3 and 4). After day 3, the survival rates were almost steady to adult stage. There was no significant difference in proportions of larvae survived to pupal stage between those from freshly laid eggs and stored eggs for 5 days across the three hatching densities $\left[\left(\chi^{2}=0.223, P=0.637\right)\right.$ at 40 eggs, $\left(\chi^{2}=0.208, P=0.648\right)$ at 80 eggs, and $\left(\chi^{2}=0.197, P=0.657\right)$ at 120 eggs (Fig. 2)]. Variation in larval survivorship was observed in larvae emerged from eggs stored for 10 days, although the difference was not statistically significant across all hatching densities $\left[\left(\chi^{2}=2.035, P=0.154\right) 40\right.$ eggs, $\left(\chi^{2}=1.407\right.$, $P=0.236) 80$ eggs, and $\left(x^{2}=2.3, P=0.129\right) 120$ eggs (Fig. 3)]. However, variation in larval survivorship was observed to be different between larvae emerged from freshly laid eggs and those stored for 15 days $\left[\left(\chi^{2}=5.249\right.\right.$, $P=0.022)$ at 40 eggs, $\left(\chi^{2}=6.211, P=0.013\right)$ at 80 eggs, and $\left(\chi^{2}=6.454, P=0.011\right)$ at 120 eggs (Fig. 4$)$ ].

\section{Comparison of sex ratios between adults from freshly laid eggs and those from stored eggs of Anopheles gambiae} The sex ratio of adult An. gambiae emerged from freshly hatched eggs across all densities was lower than 0.5 , indicating more males than females in the emerged population. The sex ratios of adult mosquitoes emerging from eggs stored for 5 and 10 days were also lower than 0.5 . However, sex ratios of adult mosquitoes emerging from eggs stored for 15 days was above 0.5 , indicating that there were more females than males. Statistical analysis revealed that sex ratios of adult mosquitoes emerging from eggs stored for 5 days across all densities were statistically higher than those from freshly hatched eggs of similar densities $(\mathrm{t}=4.99, P=0.004)$ at 40 eggs, $(\mathrm{t}=6.69, P<0.001)$ at 80 eggs, and $(\mathrm{t}=8.23, P<0.001)$ at 120 eggs densities (Fig. 5). There was significant differences between sex ratios of adult mosquitoes emerging from eggs stored for 10 days and those emerging from freshly hatched eggs at 40 eggs $(t=8.74, P<0.001)$, at 80 eggs $(\mathrm{t}=14.45, P \ll 0.001)$, and at 120 eggs $(\mathrm{t}=20.90$, $P<0.001$ ) (Fig. 6). The same was found in sex ratio of adults emerged from eggs stored for 15 days and freshly 
Table 2 The effects of storage of An. gambiae s.s. eggs on larval development time from larval to adult stage

\begin{tabular}{|c|c|c|c|c|c|c|}
\hline \multirow{3}{*}{$\begin{array}{l}\text { Eggs storage } \\
\text { period }\end{array}$} & \multirow{3}{*}{$\begin{array}{l}\text { Hatching } \\
\text { density }(n)\end{array}$} & \multicolumn{5}{|c|}{ Larval developmental time (Mean \pm SD) (days) } \\
\hline & & \multicolumn{3}{|c|}{ Duration L1 to pupal } & \multicolumn{2}{|c|}{ Duration L1 to adults } \\
\hline & & Freshly laid & Stored eggs & P values (L1 to pupal stage) & Freshly laid & Stored eggs \\
\hline \multirow[t]{3}{*}{5 days } & 40 & $7.1 \pm 1.31$ & $7.7 \pm 1.49$ & $t=1.981, P=0.12$ & $10.2 \pm 1.49$ & $10.6 \pm 0.52$ \\
\hline & 80 & $7.9 \pm 1.71$ & $8.3 \pm 1.79$ & $\mathrm{t}=2.409, \mathrm{P}=0.12$ & $12.8 \pm 1.79$ & $12.0 \pm 0.63$ \\
\hline & 120 & $8.7 \pm 1.91$ & $9.3 \pm 2.11$ & $\mathrm{t}=1.942, \mathrm{P}=0.12$ & $13.4 \pm 2.11$ & $13.7 \pm 0.81$ \\
\hline \multirow[t]{3}{*}{10 days } & 40 & $7.2 \pm 1.35$ & $7.9 \pm 1.54$ & $t=1.689, P=0.17$ & $10.9 \pm 1.54$ & $10.8 \pm 0.41$ \\
\hline & 80 & $8.0 \pm 1.72$ & $8.7 \pm 1.81$ & $t=2.361, P=0.08$ & $12.7 \pm 1.83$ & $13.5 \pm 0.55$ \\
\hline & 120 & $8.8 \pm 1.98$ & $9.4 \pm 2.23$ & $t=1.696, P=0.17$ & $13.9 \pm 2.23$ & $14.3 \pm 0.52$ \\
\hline \multirow[t]{3}{*}{15 days } & 40 & $7.3 \pm 1.42$ & $8.3 \pm 1.89$ & $t=1.086, P=0.34$ & $10.3 \pm 1.89$ & $10.0 \pm 0.63$ \\
\hline & 80 & $8.2 \pm 1.79$ & $8.9 \pm 1.94$ & $t=0.705, P=0.52$ & $12.9 \pm 1.94$ & $13.1 \pm 0.55$ \\
\hline & 120 & $8.9 \pm 2.09$ & $9.8 \pm 2.26$ & $t=0.991, P=0.38$ & $13.8 \pm 2.26$ & $13.7 \pm 0.52$ \\
\hline
\end{tabular}

Table 3 Effects of storage of An. gambiae s.s. eggs on larval survivorship from larval to adult stage

\begin{tabular}{|c|c|c|c|c|c|}
\hline \multirow[t]{3}{*}{ Eggs storage period } & \multirow[t]{3}{*}{ Hatching density (n) } & \multicolumn{4}{|c|}{ Larval survivorship (\%) } \\
\hline & & \multicolumn{2}{|c|}{ Survival I1 to pupal } & \multicolumn{2}{|c|}{ Survival L1 to pupal } \\
\hline & & Freshly laid & Stored eggs & Freshly laid & Stored eggs \\
\hline \multirow[t]{3}{*}{5 days } & 40 & $92.9 \pm 1.49$ & $91.2 \pm 1.01$ & $91.5 \pm 1.49$ & $91.2 \pm 1.01$ \\
\hline & 80 & $93.1 \pm 0.76$ & $90.6 \pm 1.14$ & $91.6 \pm 0.76$ & $90.6 \pm 1.14$ \\
\hline & 120 & $91.1 \pm 1.72$ & $89.6 \pm 1.32$ & $92.6 \pm 1.72$ & $87.6 \pm 1.43$ \\
\hline \multirow[t]{3}{*}{10 days } & 40 & $91.7 \pm 0.82$ & $78.6 \pm 1.93$ & $91.7 \pm 0.82$ & $77.3 \pm 1.04$ \\
\hline & 80 & $90.4 \pm 0.82$ & $81.6 \pm 0.97$ & $90.4 \pm 0.82$ & $81.6 \pm 0.97$ \\
\hline & 120 & $91.1 \pm 1.37$ & $79.5 \pm 1.53$ & $91.1 \pm 1.37$ & $79.5 \pm 1.53$ \\
\hline \multirow[t]{3}{*}{15 days } & 40 & $91.7 \pm 1.03^{\mathrm{dx}}$ & $18.8 \pm 1.34$ & $91.7 \pm 1.03^{\mathrm{dx}}$ & $18.8 \pm 1.34$ \\
\hline & 80 & $91.5 \pm 1.09^{d x}$ & $17.2 \pm 0.67$ & $91.5 \pm 1.09^{\mathrm{dx}}$ & $17.2 \pm 0.67$ \\
\hline & 120 & $92.2 \pm 1.03^{\mathrm{dx}}$ & $19.4 \pm 1.84$ & $92.2 \pm 1.03^{\mathrm{dx}}$ & $19.4 \pm 1.84$ \\
\hline
\end{tabular}

$\overline{(d x)}$ Letters superscripts indicates that values in respective rows are significantly different at $P<0.05$ by Kaplan-Meier test

hatched eggs $(\mathrm{t}=19.08, P<0.001)$ at 40 eggs, $(\mathrm{t}=19.36$, $P<0.001)$ at 80 eggs, and $(\mathrm{t}=19.23, P<0.001)$ at 120 eggs (Fig. 7).

\section{Discussion}

The current study underlays a foundation for An. gambiae egg storage effect on egg viability, larvae development and emerged adult sex ratio which is crucial information on the mass rearing of mosquitoes. The aim of the present study was to investigate the effects of storing $A n$. gambiae eggs at $-20{ }^{\circ} \mathrm{C}$ for $10 \mathrm{~min}$ and then transferring to $4{ }^{\circ} \mathrm{C}$ and $48 \pm 2 \% \mathrm{RH}$ on hatchability, larval development, larval survivorship, and emerged adult sex ratios. It was found that storage time had negative consequences on egg hatchability, larval development rates and larval survivorship as number of storage days increased. Additionally, there was skewed sex ratio as adult females increased as egg storage time increased.
These findings show that egg hatchability patterns decreased with increased time of egg storage; 93 and $85 \%$ hatch rates were recorded at 5 and 10 storage days, respectively. This pattern was similar to studies conducted by Khan and others in which egg hatch rates were reported to decrease with increase of egg storage time at $16{ }^{\circ} \mathrm{C}$ [23]. Relatively high hatch rates were also reported in $A n$. gambiae that were stored at $12{ }^{\circ} \mathrm{C}$ for 1 and 3 days, with reduction in hatch rate observed for eggs stored for longer, for 7 and 10 days [31]. Embryonic development has been recognized to be highly influenced by external stimuli particularly temperature [31]. It has been shown that exposure of mosquito eggs to temperature below the optimal threshold $\left(24{ }^{\circ} \mathrm{C}\right)$ may lead to diapauses [32]. It follows that prolonged exposure in diapause state depletes embryological resources and consequently results in embryo mortality [31]. Brown and others have reported a decrease in hatch rate with an increase in storage time in Aedes 


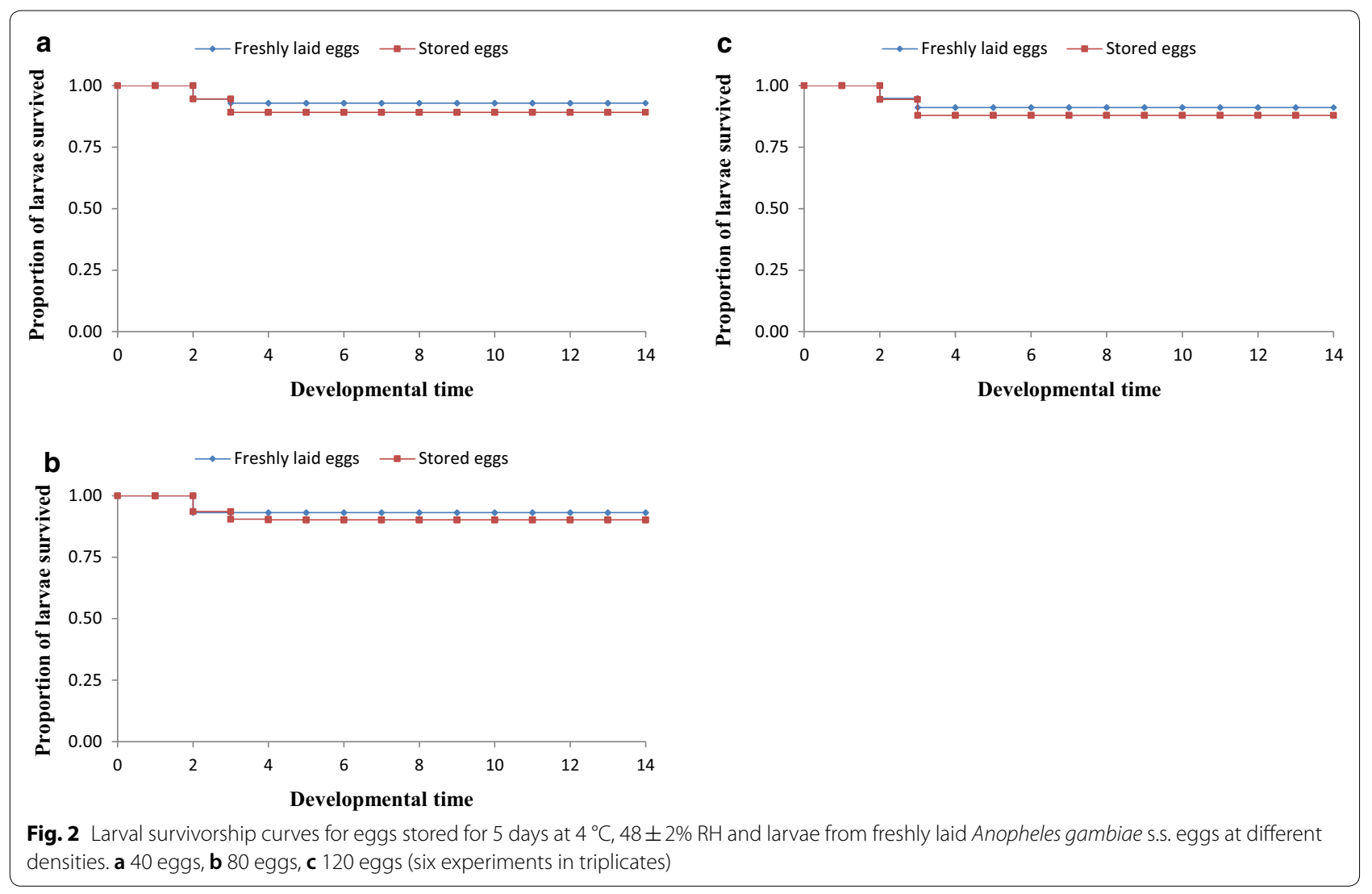

aegypti, i.e., for every added day of Aedes aegypti egg incubation at $26.5{ }^{\circ} \mathrm{C}$, hatch rates dropped by $0.27 \%$ [33]. Hatch rate index (HRI) negatively associates with time of egg exposure into diapauses. Since storing eggs for 10 days resulted in significantly lower hatch rate compared to freshly laid eggs in this study, storing eggs for 5 days at $4{ }^{\circ} \mathrm{C}$ and $48 \pm 2 \% \mathrm{RH}$ is considered optimal duration as it maintained higher egg hatchability compared to freshly laid eggs.

The effect of egg storage on larval development has shown that An. gambiae larval development was inversely proportional to storage time at all densities although the difference was not significant. The findings of this study have been found similar to previous reported a delayed An. gambiae and Aedes aegypti larval development with increased egg storage time [23, 31]. Reduced locomotor and feeding activities have been reported in diapause-terminated larvae [34]. Several studies have reported a strong shift in gene expression patterns and regulatory pathways during diapauses $[35,36]$. The shift in gene expression patterns may lead to lack of normal physiological development. Delayed larval development observed in larvae from stored eggs in the present study might have resulted from impacts of diapauses and changes in gene expression patterns, as previously reported in various studies [23, 31]. The evidence shows that the rate of insect larvae growth and development gradually diminishes as the exposure of micro-embryo to a lower temperature threshold increases [37, 38]. According to Beck-Johnson et al. when insect eggs are subjected to lower temperature thresholds, micro-embryo physiological processes, including development, are retarded or sometimes ceased [39]. Physiological processes are regained when developing embryos are subjected to normal temperature thresholds, although retarded effects have been shown to determine subsequent developmental parameters [40]. In the present study, larvae that emerged from eggs stored for 5 days at $4{ }^{\circ} \mathrm{C}$ and $48 \pm 2 \% \mathrm{RH}$ took less time (8.4 days) to pupate than larvae from eggs stored for 10 and 15 days (8.6 and 9 days, respectively). That is, the pupation period increased with increased time of egg storage. Larval development trends observed in this study are likely similar to previous studies on An. arabiensis and Culex quinquefasciatus, in which larval developmental time increased relatively to increase in storage time $[23,41]$. The delayed larval development observed in the present study might have been induced by 


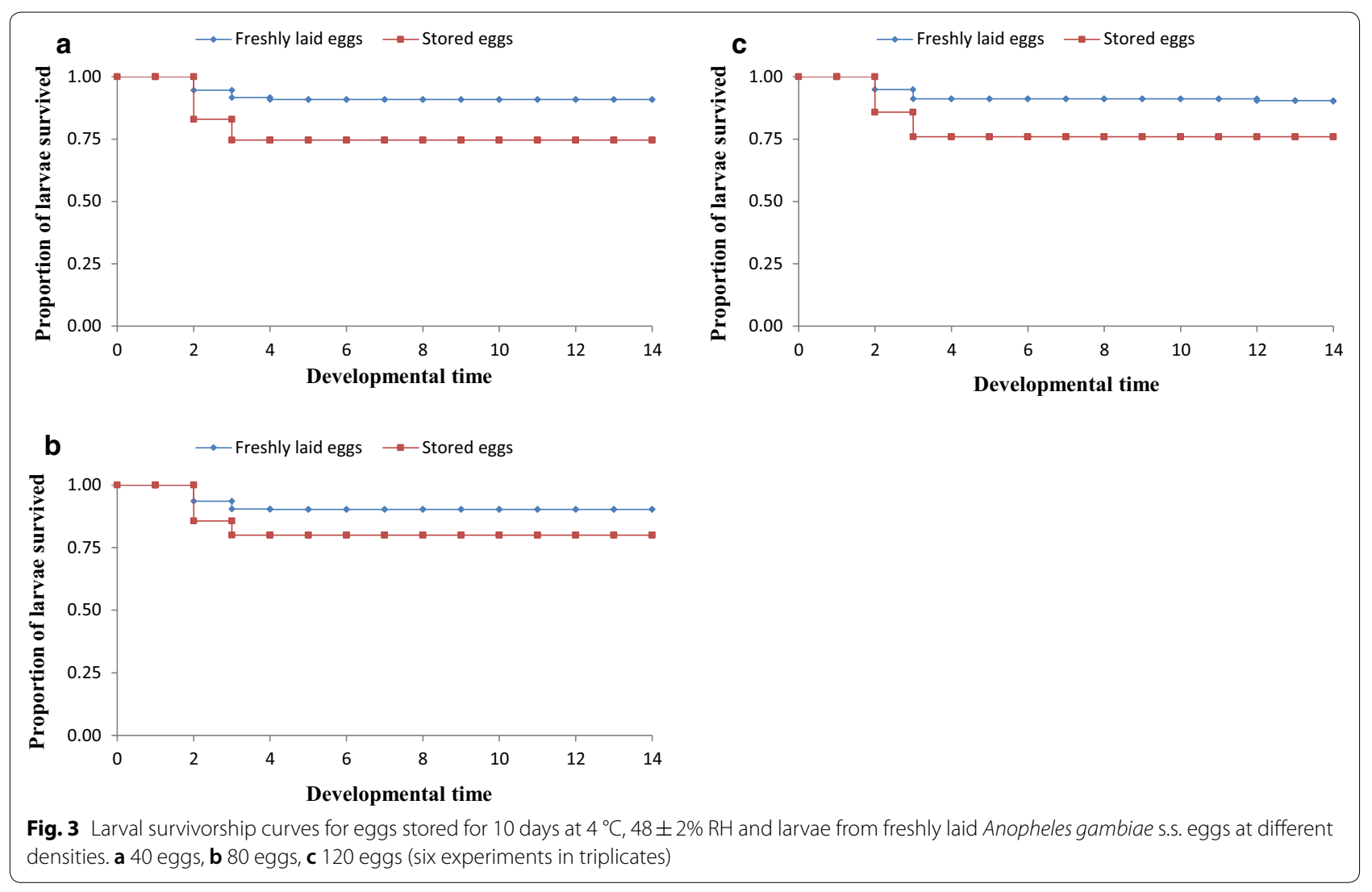

thermal effects of storage temperature $\left(4{ }^{\circ} \mathrm{C}, 48 \pm 2 \% \mathrm{RH}\right)$ and increased time of storage on embryogenesis.

This study revealed that increase in storage time had negative consequences on larval survivorship. This means that larvae mortality was inversely proportional to egg storage time. Higher larval mortalities were recorded in eggs stored for 15 days. In insectary practices it is known that when mosquito eggs are stored in humid conditions, embryos may mature before eggs are washed into hatching plates [42, 43]. Longer exposure periods in diapauses before hatching were likely to affect larval survivorship as food and energy are exhausted before hatch. Similar to this study are findings from Russell and others who observed a decline in Ae. aegypti larvae survivorship with an increase in storage time at $25-26^{\circ} \mathrm{C}$ and $95 \% \mathrm{RH}$ [44]. This suggests that storing eggs might have made the larvae too feeble to survive soon after hatch as has been shown in the present study and previous studies $[45,46]$.

The results from this study have several practical applications. When An. gambiae eggs are stored in moistened condition at lower temperatures they can still be utilized for seeding collection. At $4{ }^{\circ} \mathrm{C}, 48 \pm 2 \% \mathrm{RH}$, An. gambiae eggs may be kept for 5 and 10 days with an anticipated hatch of 93 and 85\%, respectively, and with no effect on rate of survivorship of emerging larvae. The ability to store Anopheles eggs for a period of time at stated conditions in this study permits flexibility in accommodating holidays or absence of personnel for mass rearing of mosquitoes.

The emerged sex ratio of eggs in this study was found to be affected by storage days. Dipterans larval sexes, particularly mosquitoes, are known to exist in either heterozygous or homozygous of male and female and expression of sexes are known to be thermally affected based on the range of temperature to which developing embryo and larvae are subjected $[47,48]$. In natural populations, males outnumbers female mosquitoes $[49,50]$. In the present study, the proportions of adult An. gambiae females were lower than males in both freshly laid eggs and those eggs stored from 5 to 10 days. Proportions of males to females in this study were skewed relatively to time the eggs were stored. Insect sex determination, particularly in dipterans, are said to be accidental, highly induced by temperature and controlled by sex-specific lethality chemicals $[50,51]$. For example, long exposure of Aedes aegypti developing embryo and larvae to lower and higher temperatures (below $3{ }^{\circ} \mathrm{C}$ or above $29^{\circ} \mathrm{C}$ ) of that of their original collection $[48,52]\left(5-20^{\circ} \mathrm{C}\right)$ was observed to induce female genital appendages to males, and which morphologically appeared to be females [48, 


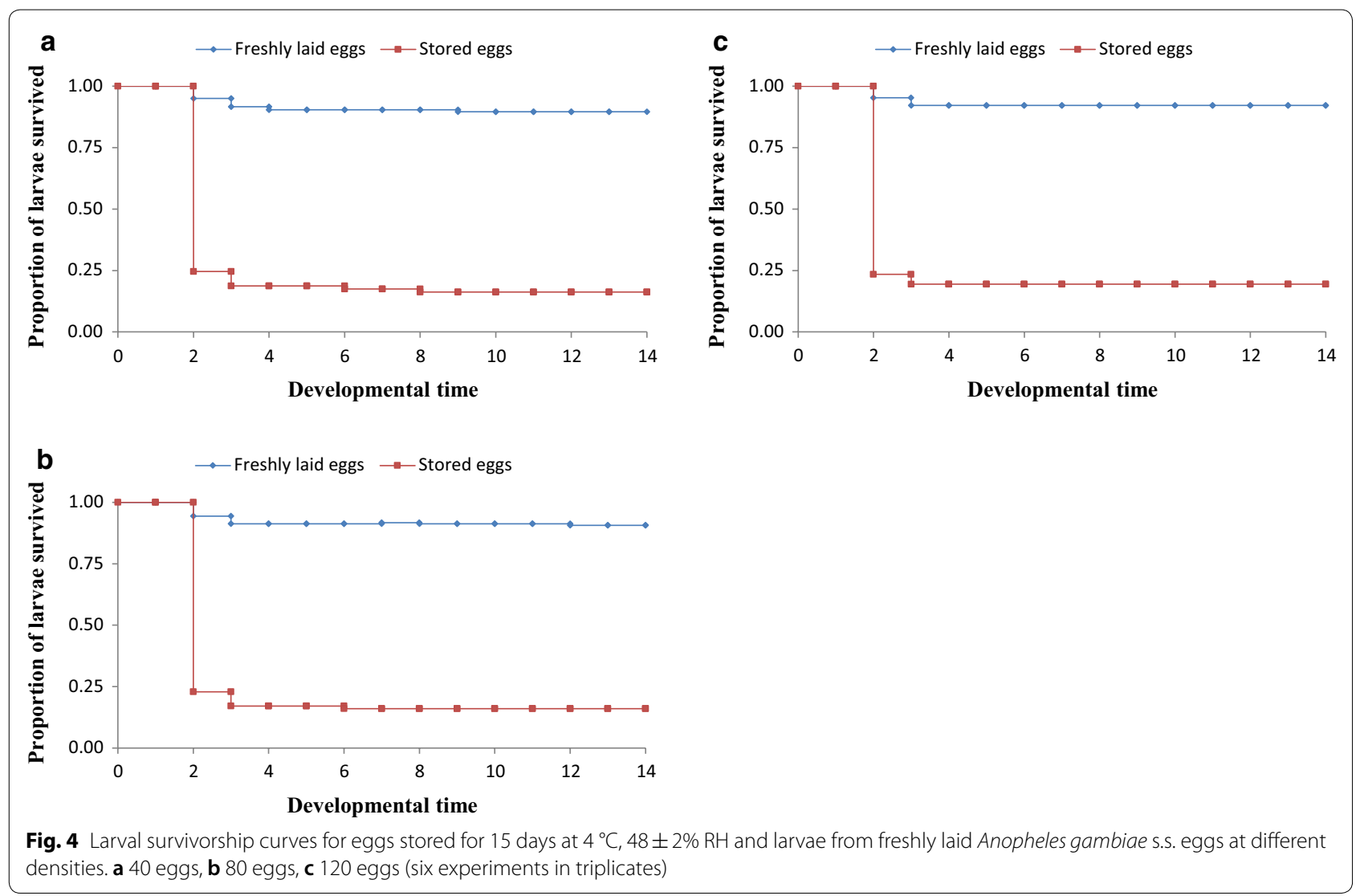

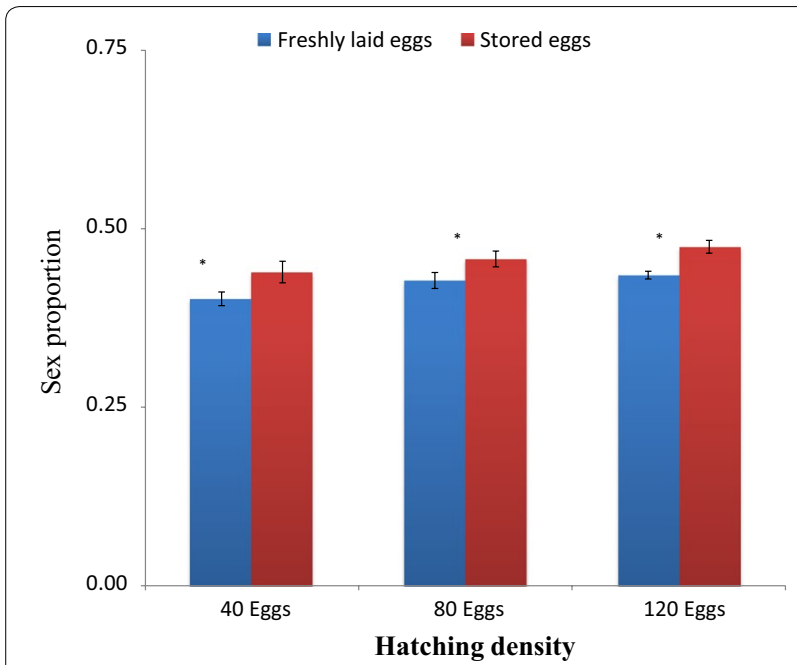

Fig. 5 Sex ratio of adult Anopheles gambiae s.s. (females emerged against total mosquitoes) (males and females emerged) from freshly laid eggs and eggs stored at $4{ }^{\circ} \mathrm{C}$ and $48 \pm 2 \% \mathrm{RH}$ for 5 days and hatched at different densities. Asterisk indicates a significant difference at $P<0.05$ by paired sampled test. Six experiments in triplicates
52]. It was also suggested that prolonged exposure of juvenile stages to higher or lower temperatures decreased dimorphism in sex determination, female sex expressivity in heterogametic insects and male sex aberration [47, 51]. Increased females and decreased males relatively to increased egg storage time observed in the present study might have been induced by effects of prolonged egg storage at $4{ }^{\circ} \mathrm{C}$ temperature. The temperature $\left(4{ }^{\circ} \mathrm{C}\right.$, $48 \pm 2 \% \mathrm{RH}$ ) might have more positive effects on female sexual expressivity than on males.

The findings of the present study can be considered to be of significance in vector and malaria infection control. It is clear that $4{ }^{\circ} \mathrm{C}, 48 \pm 2 \% \mathrm{RH}$ can be used as the temperature to store Anopheles eggs in mass rearing of An. gambiae under insectary conditions without significantly affecting adult sex ratio. Since there were higher numbers of emerged males than females from eggs that were stored for 5 days $\left(4{ }^{\circ} \mathrm{C}, 48 \pm 2 \% \mathrm{RH}\right)$ compared to eggs stored for 10 and 15 days $\left(4{ }^{\circ} \mathrm{C}, 48 \pm 2 \% \mathrm{RH}\right)$, and since sterile insect technique requires only male mosquitoes for sterilization, 5 days at $4{ }^{\circ} \mathrm{C}$ and $48 \pm 2 \% \mathrm{RH}$ 


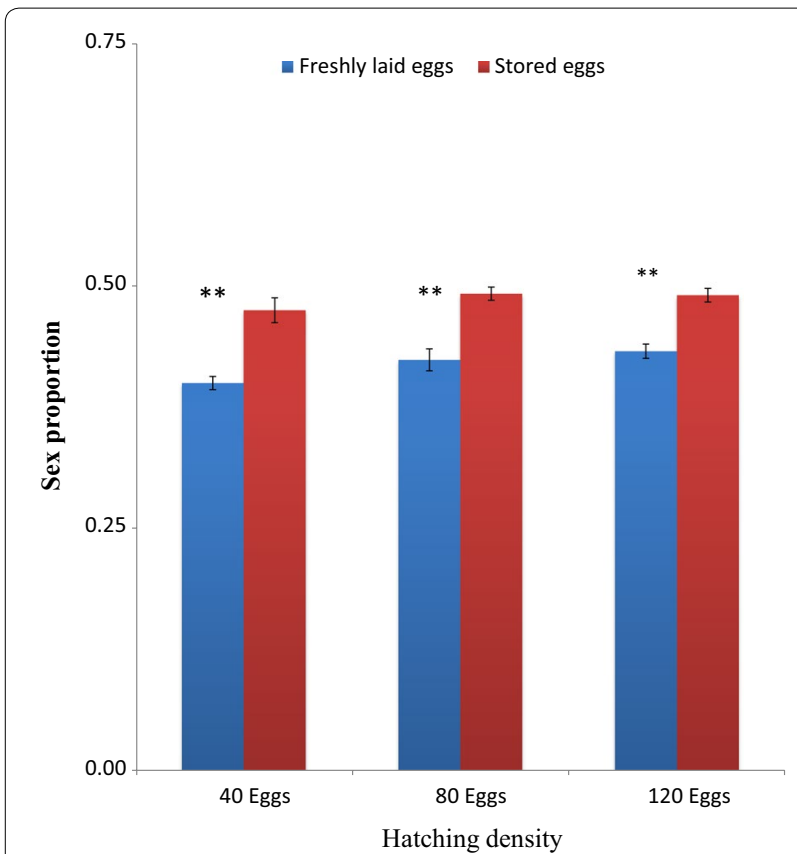

Fig. 6 Sex ratio of adult Anopheles gambiae s.s. (females emerged against total mosquitoes) (males and females emerged) from freshly laid eggs and eggs stored at $4{ }^{\circ} \mathrm{C}$ and $48 \pm 2 \%$ RH for 10 days and hatched at different densities. Double asterisk indicates a significant difference at $P<0.001$ by paired sampled test. Six experiments in triplicates

can be considered the optimal duration and condition for storing An. gambiae eggs to be used in mass rearing for sterile insect technique.

Overall, the present study has shown that storing $A n$. gambiae eggs for 5 days at $4{ }^{\circ} \mathrm{C}$ is an optimal condition for storage with no significant effects on emerged adults sex ratios. This storage condition did not affect An. gambiae egg hatch rates, larval development and larval survivorship and, therefore, this condition can be used in storage of anopheline eggs for a period of time to permit flexibility in accommodating the routine or absence of personnel involved in mass rearing of mosquitoes. The study may also be important in establishing a protocol for raising male mosquitoes in sterile insect technique.

\section{Conclusion}

Findings of this present study can be used for information on storage conditions of anopheline eggs as well as the establishment of mass rearing protocols for An. gambiae mosquitoes. More research is needed to explore emerged adult survival characteristics, such as mating competitiveness, response to insecticide exposure, vector

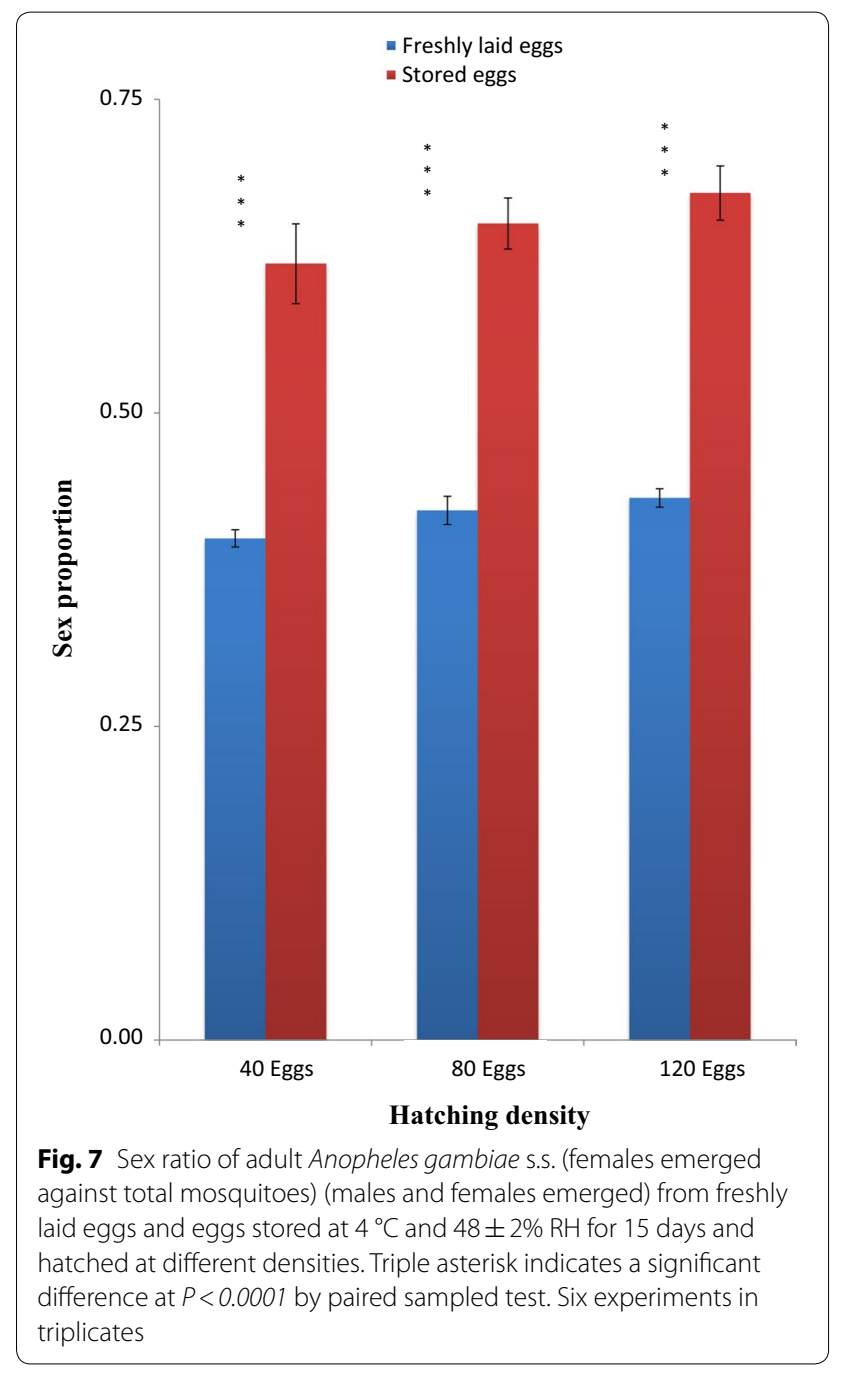

competence as well as molecular and genetic status of reared adult mosquitoes to determine whether the proposed storage temperature and storage time induced any molecular variations.

\section{Authors' contributions}

EM, WK, JM, and EJK contributed to study conceived and design. EM and WK coordinated experiments. EM undertook laboratory work. EM and EJK analysed data. EM, WK, JM, and EJK drafted the manuscript. All authors read and approved the final manuscript.

\section{Author details}

${ }^{1}$ Department of Zoology and Wild life Conservation, College of Natural and Applied Sciences, University of Dar es Salaam, Dar es Salaam, Tanzania.

${ }^{2}$ National Institute for Medical Research, Amani Medical Research Centre, P.O. Box 81, Muheza, Tanzania. ${ }^{3}$ Department of Medical Parasitology, School of Medicine, Catholic University of Health and Allied Sciences-Bugando, P.O. Box 1464, Mwanza, Tanzania. ${ }^{4}$ Division of Livestock and Human Disease Vector Control, Tropical Pesticides Research Institute, P.O.Box 3024, Arusha, Tanzania. 


\section{Acknowledgements}

Authors are thankful to Mr. Massawe and Ms. Germima of TPRI who assisted during insectary works. Staffs of TPRI are acknowledged for supporting this study with critical comments and inputs during this study progress. This study had no financial support but TPRI infrastructure supported the study.

\section{Competing interests}

The authors declare that they have no competing interests.

\section{Availability of data and materials}

The datasets used and/or analysed during the current study are available from the corresponding author on reasonable request.

\section{Consent for publication}

Not applicable.

\section{Ethics approval and consent to participate}

The protocol of this study was reviewed and approved by the ethical review board of university of Dar-es-Salaam.

\section{Funding}

This study had no financial support from any source. It used available resources from TPRI insectary.

\section{Publisher's Note}

Springer Nature remains neutral with regard to jurisdictional claims in published maps and institutional affiliations.

Received: 10 December 2018 Accepted: 21 February 2019 Published online: 26 February 2019

\section{References}

1. Coetzee M, Hunt RH, Wilkerson R, Della Torre A, Coulibaly MB, Besansky NJ. Anopheles coluzzii and Anopheles amharicus, new members of the Anopheles gambiae complex. Zootaxa. 2013;3619:246-74.

2. Sinka ME, Rubio-Palis Y, Manguin S, Patil AP, Temperley WH, Gething PW, et al. The dominant Anopheles vectors of human malaria in the Americas: occurrence data, distribution maps and bionomic précis. Parasit Vectors. 2010;3:72.

3. WHO. World malaria report 2018. Geneva: World Health Organization; 2018

4. O'Loughlin SM, Magesa SM, Mbogo C, Mosha F, Midega J, Burt A. Genomic signatures of population decline in the malaria mosquito Anopheles gambiae. Malar J. 2016;15:182.

5. Chinula D, Hamainza B, Chizema E, Kavishe DR, Sikaala CH, Killeen GF. Proportional decline of Anopheles quadriannulatus and increased contribution of An. arabiensis to the An. gambiae complex following introduction of indoor residual spraying with pirimiphos-methyl: an observational, retrospective secondary analysis of pre-existing data from south-east Zambia. Parasit Vectors. 2018;11:544.

6. Alegana VA, Kigozi SP, Nankabirwa J, Arinaitwe E, Kigozi R, Mawejje H, et al. Spatio-temporal analysis of malaria vector density from baseline through intervention in a high transmission setting. Parasit Vectors. 2016;9:637.

7. Sy O, Niang EHA, Ndiaye M, Konaté L, Diallo A, Ba ECC, et al. Entomological impact of indoor residual spraying with pirimiphos-methyl: a pilot study in an area of low malaria transmission in Senegal. Malar J. 2018;17:64.

8. Thwing J, Eckert E, Dione DA, Tine R, Faye A, Yé Y, Ndiop M, et al. Declines in malaria burden and all-cause child mortality following increases in control interventions in Senegal, 2005-2010. Am J Trop Med Hyg. 2017:97:89-98.

9. WHO. World malaria report 2017. Geneva: World Health Organization; 2018.

10. WHO. Action and investment to defeat malaria 2016-2030-for a malariafree world, Technical report. Geneva: World Health Organization; 2016.
11. Gnanguenon V, Agossa FR, Badirou K, Govoetchan R, Anagonou R, OkeAgbo F, et al. Malaria vectors resistance to insecticides in Benin: current trends and mechanisms involved. Parasit Vectors. 2015;8:223.

12. Protopopoff N, Matowo J, Malima R, Kavishe R, Kaaya R, Wright A, et al. High level of resistance in the mosquito Anopheles gambiae to pyrethroid insecticides and reduced susceptibility to bendiocarb in north-western Tanzania. Malar J. 2013;12:149.

13. Rakotoson J-D, Fornadel CM, Belemvire A, Norris LC, George K, Caranci A, et al. Insecticide resistance status of three malaria vectors, Anopheles gambiae (s.l.), An. funestus and An. mascarensis, from the south, central and east coasts of Madagascar. Parasit Vectors. 2017;10:396.

14. Ranson H, N'guessan R, Lines J, Moiroux N, Nkuni Z, Corbel V. Pyrethroid resistance in African anopheline mosquitoes: what are the implications for malaria control? Trends Parasitol. 2011:27:91-8.

15. Vulule J, Beach R, Atieli F, Roberts J, Mount D, Mwangi R. Reduced susceptibility of Anopheles gambiae to permethrin associated with the use of permethrin-impregnated bednets and curtains in Kenya. Med Vet Entomol. 1994;8:71-5.

16. Dambach P, Schleicher M, Korir P, Ouedraogo S, Dambach J, Sié A, et al. Nightly biting cycles of Anopheles species in rural Northwestern Burkina Faso. J Med Entomol. 2018;55:1027-34.

17. Padonou GG, Sezonlin M, Ossé R, Aizoun N, Oké-Agbo F, Oussou O, et al. Impact of three years of large scale Indoor Residual Spraying (IRS) and Insecticide Treated Nets (ITNs) interventions on insecticide resistance in Anopheles gambiae s.l. in Benin. Parasit Vectors. 2012;5:72.

18. Asale A, Duchateau L, Devleesschauwer B, Huisman G, Yewhalaw D. Zooprophylaxis as a control strategy for malaria caused by the vector Anopheles arabiensis (Diptera: Culicidae): a systematic review. Infect Dis Poverty. 2017;6:160.

19. Gatton ML, Chitnis N, Churcher T, Donnelly MJ, Ghani AC, Godfray HC, et al. The importance of mosquito behavioural adaptations to malaria control in Africa. Evolution. 2013;67:1218-30.

20. WHO. World health statistics. monitoring health for the SDGs sustainable development goals. Geneva: World Health Organization; 2016. p. 2017.

21. WHO-UNICEF. Assessment of research needs for public health adaptation to social, environmental and climate change impacts on vector-borne diseases in Africa., An informal expert consultation convened by the Special Programme for Research and Training in Tropical Diseases (TDR), Addis Ababa, Ethiopia, 2012Geneva: World Health Organization; 2012.

22. Bouyer J, Lefrançois T. Boosting the sterile insect technique to control mosquitoes. Trend Parasitol. 2014;30:271-3.

23. Khan I, Damiens D, Soliban SM, Gilles JR. Effects of drying eggs and egg storage on hatchability and development of Anopheles arabiensis. Malar J. 2013:12:318.

24. Nolan T, Papathanos P, Windbichler N, Magnusson K, Benton J, Catteruccia F, et al. Developing transgenic Anopheles mosquitoes for the sterile insect technique. Genetica. 2011;139:33-9.

25. Beier J, Copeland R, Oyaro C, Masinya A, Odago W, Oduor S, et al. Anopheles gambiae complex egg-stage survival in dry soil from larval development sites in western Kenya. J Am Mosq Control Assoc. 1990;6:105-9.

26. Yaro AS, Dao A, Adamou A, Crawford JE, Ribeiro JMC, Gwadz R, et al. The distribution of hatching time in Anopheles gambiae. Malar J. 2006;5:19.

27. Soares-Pinheiro V, Dasso-Pinheiro W, Trindade-Bezerra J, Tadei WP. Eggs viability of Aedes aegypti Linnaeus (Diptera, Culicidae) under different environmental and storage conditions in Manaus, Amazonas, Brazil. Braz J Biol. 2017;77:396-401.

28. Ebrahimi B, Shakibi S, Foster WA. Delayed egg hatching of Anopheles gambiae (Diptera: Culicidae) pending water agitation. J Med Entomol. 2014:51:580-90.

29. World Health Organization. Manual on practical entomology in malaria. Part II. Methods and techniques. Geneva: World Health Organization; 1975.

30. Kivuyo HS, Mbazi PH, Kisika DS, Munga S, Rumisha SF, Urasa FM, et al. Performance of five food regimes on Anopheles gambiae senso stricto larval rearing to adult emergence in insectary. PLoS ONE. 2014;9:e110671.

31. Impoinvil DE, Cardenas GA, Gihture Jl, Mbogo CM, Beier JC. Constant temperature and time period effects on Anopheles gambiae egg hatching. J Am Mosq Control Assoc. 2007;23:124-30.

32. Judson CL, Hokama Y, Haydock I. The physiology of hatching of aedine mosquito eggs: hatching stimulus. J Insect Physiol. 1965;11:1169-77. 
33. Brown HE, Smith C, Lashway S. Influence of the length of storage on Aedes aegypti (Diptera: Culicidae) egg viability. J Med Entomol. 2017:54:489-91.

34. Mori A, Oda T, Wada Y. Studies on the egg diapause and overwintering of Aedes albopictus in Nagasaki. Trop Med. 1981;23:79-90.

35. Poelchau MF, Reynolds JA, Elsik CG, Denlinger DL, Armbruster PA. Deep sequencing reveals complex mechanisms of diapause preparation in the invasive mosquito, Aedes albopictus. Proc Biol Sci. 2013;280:20130143.

36. Urbanski J, Mogi M, O'Donnell D, DeCotiis M, Toma T, Armbruster P. Rapid adaptive evolution of photoperiodic response during invasion and range expansion across a climatic gradient. Am Nat. 2012;179:490-500.

37. Carvalho SC, Martins AJ, Lima JBP, Valle D. Temperature influence on embryonic development of Anopheles albitarsis and Anopheles aquasalis. Mem Inst Oswaldo Cruz. 2002;97:1117-20.

38. Howe RW, Currie JE. Some laboratory observations on the rates of development, mortality and oviposition of several species of Bruchidae breeding in stored pulses. Bull Entomol Res. 1964;55:437-77.

39. Beck-Johnson LM, Nelson WA, Paaijmans KP, Read AF, Thomas MB, Bjornstad ON. The effect of temperature on Anopheles mosquito population dynamics and the potential for malaria transmission. PLoS ONE. 2013;8:e79276.

40. Koenraadt CJ, Paaijmans KP, Githeko AK, Knols BG, Takken W. Egg hatching, larval movement and larval survival of the malaria vector Anopheles gambiae in desiccating habitats. Malar J. 2003;2:20.

41. Shriver D, Bickley W. The effect of temperature on hatching of eggs of the mosquito, Culex pipiens quinquefasciatus Say. Mosq News. 1964:24:137-40

42. Briegel H, Gut T, Lea AO. Sequential deposition of yolk components during oogenesis in an insect, Aedes aegypti (Diptera: Culicidae). J Insect Physiol. 2003;49:249-60.
43. Briegel H, Timmermann SE. Aedes albopictus (Diptera: Culicidae): physiological aspects of development and reproduction. J Med Entomol. 2001;38:566-71.

44. Russell B, Kay B, Shipton W. Survival of Aedes aegypti (Diptera: Culicidae) eggs in surface and subterranean breeding sites during the northern Queensland dry season. J Med Entomol. 2001;38:441-5.

45. Faull KJ, Williams CR. Intraspecific variation in desiccation survival time of Aedes aegypti (L.) mosquito eggs of Australian origin. J Vector Ecol. 2015:40:292-300

46. Meola R. The influence of temperature and humidity on embryonic longevity in Aedes aegypti. Ann Entomol Soc Am. 1964;57:468-72.

47. Horsfall WR, Anderson JF. Suppression of male characteristics of mosquitoes by thermal means. Science. 1961;133:1830.

48. Horsfall WR, Anderson JF. Thermally induced genital appendages on mosquitoes. Science. 1963;141:1183-4.

49. Kweka EJ, Zhou G, Beilhe LB, Dixit A, Afrane Y, Gilbreath TM, et al. Effects of co-habitation between Anopheles gambiae s.s. and Culex quinquefasciatus aquatic stages on life history traits. Parasit Vectors. 2012;5:33.

50. Mutuku FM, Bayoh MN, Gimnig JE, Vulule JM, Kamau L, Walker ED, et al. Pupal habitat productivity of Anopheles gambiae complex mosquitoes in a rural village in western Kenya. Am J Trop Med Hyg. 2006;74:54-61.

51. Kageyama D, Narita S, Watanabe M. Insect sex determination manipulated by their endosymbionts: incidences, mechanisms and implications. Insects. 2012;3:161-99.

52. Brust R. Temperature-induced intersexes in Aedes mosquitoes: comparative study of species from Manitoba. Can Entomol. 1968;100:879-91.
Ready to submit your research? Choose BMC and benefit from:

- fast, convenient online submission

- thorough peer review by experienced researchers in your field

- rapid publication on acceptance

- support for research data, including large and complex data types

- gold Open Access which fosters wider collaboration and increased citations

- maximum visibility for your research: over $100 \mathrm{M}$ website views per year

At BMC, research is always in progress.

Learn more biomedcentral.com/submissions 